\title{
Efficacy of small-dose ganciclovir on cytomegalovirus infections in children and its effects on liver function and miR-UL112-3p expression
}

\author{
QINGXIU WANG $^{1 *}$, WENZENG ZHOU ${ }^{2 *}$, BIN WANG $^{3}$, GUOYUN QIN $^{4}$, \\ FENG'AI LIU ${ }^{5}$, DEXIANG LIU ${ }^{6}$ and TENGTENG HAN ${ }^{2}$
}

\begin{abstract}
${ }^{1}$ Office of Hospital Infection Management, Affiliated Hospital of Weifang Medical University, Weifang, Shandong 261031;
${ }^{2}$ Department of Child Rehabilitation, Zaozhuang Maternal and Child Health Hospital of Shandong Province,

Zaozhuang, Shandong 277100; ${ }^{3}$ Department of Child Rehabilitation, The Second People's Hospital of Liaocheng, Liaocheng, Shandong 252600; ${ }^{4}$ Department of Pharmacy, Yidu Central Hospital, Weifang, Shandong

262500; ${ }^{5}$ Department of Paediatrics, Haiyang People's Hospital of Shandong Province, Haiyang, Shandong

265100; ${ }^{6}$ Department of Pediatrics, Laoling People's Hospital, Laoling, Shandong 253600, P.R. China
\end{abstract}

Received April 3, 2020; Accepted February 1, 2021

DOI: $10.3892 / \mathrm{etm} .2021 .10344$

\begin{abstract}
The aim of the study was to explore the efficacy of small-dose ganciclovir on cytomegalovirus infections as well as its effects on the liver function and miR-UL112-3p of children. A total of 141 children infected with cytomegalovirus admitted to the Affiliated Hospital of Weifang Medical University from May 2015 to August 2017 were enrolled, of which 74 children were treated with small-dose ganciclovir as an observation group (Obs group), and the rest were treated with conventional-dose ganciclovir as a control group (Con group). The two groups were compared in efficacy after treatment, changes of liver function indexes [total bilirubin (TB), alanine aminotransferase (ALT), and aspartate aminotransferase (AST)] and miR-UL112-3p before and after treatment, and adverse reactions after treatment. A receiver operating characteristic (ROC) curve was drawn to analyze the value of miR-UL112-3p in predicating efficacy on cytomegalovirus infections in children, and Pearson's correlation analysis was carried out to analyze the correlation between miR-UL112-3p expression and TB, ALT and AST. The MV-DNA level between the two groups after treatment was compared. The two groups showed no significant difference in efficacy and adverse reactions (both $\mathrm{P}>0.05$ ), and before
\end{abstract}

Correspondence to: Dr Tengteng Han, Department of Child Rehabilitation, Zaozhuang Maternal and Child Health Hospital of Shandong Province, 25 Wenhua East Road, Shizhong, Zaozhuang, Shandong 277100, P.R. China

E-mail: isqsg8@163.com

*Contributed equally

Key words: ganciclovir, small dose, cytomegalovirus infections in children, liver function, miR-UL112-3p treatment, there was also no significant difference between the two groups in miR-UL112-3p, TB, ALT, and AST, while after treatment, both groups showed lower levels of miR-UL112-3p, TB, ALT, and AST, and the Obs group showed significantly lower levels thereof than the Con group (all $\mathrm{P}<0.05$ ). In addition, the area under the curve (AUC), specificity, and sensitivity of miR-UL112-3p in the ROC curve of the Obs group were $0.866,73.77$ and $84.62 \%$, respectively, while the AUC, specificity, and sensitivity of the ROC of the Con group were 0.837 , 75.44, and $90.00 \%$, respectively. Furthermore, miR-UL112-3p was positively correlated with TB, ALT, and AST, respectively. The CMV-DNA level in the Obs group was lower than that in the Con group, but the difference was not significant, and the level of CMV-DNA was positively correlated with that of miR-UL112-3p. In conclusion, small-dose ganciclovir can better improve the liver function of the children, and downregulate miR-UL112-3p in them. The AUC, specificity, and sensitivity of miR-UL112-3p for predicting the efficacy of small-dose ganciclovir were $0.866,73.77$ and $84.62 \%$, respectively, and the AUC, specificity, and sensitivityfor predicting the efficacy of conventional-dose ganciclovir were 0.837 , 75.44 and $90.00 \%$, respectively.

\section{Introduction}

Human cytomegalovirus is a herpesvirus, which is widespread in people, and most infected individuals exhibit no specific clinical characteristics. According to statistics, the infection rate of this virus in adults in the West is between 60 and $70 \%$ (1). However, children are the main vulnerable group of the virus. The body function of children is not fully developed and their immune function is weaker than that of normal adults, suggesting that more attention should be paid to children infected with cytomegalovirus with regard to diagnosis and treatment (2). Cytomegalovirus often leads to pathological changes in the lung and liver of children, and its infection is one of the primary risk factors for hearing damage in children, which can lead to 
dysplasia of brain and neuron, and even death (3-5). Therefore, it is imperative to evaluate the severity of illness and efficacy on children and to implement effective treatments.

MicroRNA is a non-coding single-stranded small RNA molecule in human body, which affects the development and progression of some diseases and can be used as a potential indicator of various diseases (6-8). Previous findings have shown that there are some exogenous microRNAs stemming from viruses and microorganisms in the human body that participate in the development and progression of some diseases (9). miR-UL112-3p is a microRNA encoded by human cytomegalovirus, which is involved in viral transcriptional activation and immune evasion, and is related to the regulation of cell cycle, proliferation and apoptosis (10). One study by Pan et al (11) has found another microRNA encoded by cytomegalovirus that may be used as a therapeutic indicator for hepatitis B. Therefore, we suspected that miR-UL112-3p can also be used to evaluate the efficacy on children.

Ganciclovir is a common antiviral drug in clinical practice, with relatively good efficacy on cytomegalovirus infection in children (12). However, there are some side effects in the treatment process, causing symptoms such as myelosuppression (13). The liver function of children infected with cytomegalovirus is often damaged. However, findings of recent studies have shown that ganciclovir can improve the liver function of patients with hepatitis $\mathrm{C}$ virus (HCV) liver fibrosis, and can alleviate hepatitis Caused by cytomegalovirus infections in children $(14,15)$. However, few studies are available on the effect of small-dose ganciclovir on the liver function of children infected with cytomegalovirus, the specific efficacy and safety thereof. and its effects on miR-UL112-3p.

Therefore, the aim of the current study was to examine the efficacy of small-dose ganciclovir and conventional-dose ganciclovir on children infected with cytomegalovirus, and analyze the changes of the liver function and miR-UL112-3p expression in children before and after treatment, to provide a basis and direction for the clinical application of ganciclovir.

\section{Materials and methods}

Patients. A total of 141 children infected with cytomegalovirus admitted to the Affiliated Hospital of Weifang Medical University from May 2015 to August 2017 were enrolled, of which 74 children were treated with small-dose ganciclovir as an observation group (Obs group), and the rest were treated with conventional-dose ganciclovir as the control group (Con group). The Obs group comprised 43 males and 31 females, with an average age of $(3.15 \pm 0.85)$ years, and the Con group consisted of 38 males and 29 females, with an average age of $(3.06 \pm 0.72)$ years.

This study was approved by the Medical Ethics Committee of the Affiliated Hospital of Weifang Medical University (SD-2015-265), and written informed consent was obtained from legal guardiansand/or parent(s) of the children after the guardians understood the study.

Inclusion and exclusion criteria. The inclusion criteria of the study were: i) Patients confirmed with cytomegalovirus infections according to virology and pathology, ii) patients $<18$ years old, iii) patients without allergic reaction to drugs used in this study, iv) patients with detailed clinical data, and v) patients willing to receive the therapy and follow-up.

The exclusion criteria included: i) Patients who had received antiviral treatment within one month before admission, ii) patients with other comorbid liver or kidney diseases, or other viral infectious diseases, and iii) patients with congenital immune diseases.

Main kits and instruments. PCR instrument (Applied Biosystems 7500), electrophoresis apparatus (Bio-Rad Mini-PROTEAN), ultraviolet spectrophotometer (Beckman Coulter, DU 700), fully automatic biochemical analyser (Abbott Aeroset 2000), total RNA extraction kit (EasyPure miRNA Kit) and reverse transcription + PCR kit (TransScript miRNA First-Strand cDNA Synthesis SuperMix; TransGen Biotech Co., Ltd.; nos. ER601-01, AT351-01). Primers were designed and synthesized by Shanghai Sangon Biotech Co., Ltd. (Table I).

Treatment methods. Children in the Obs group were treated with small-dose ganciclovir as follows: They were given ganciclovir mixed with $5 \%$ glucose solution through intravenous drip at $3 \mathrm{mg} / \mathrm{kg}$ (12 h/time) for a course of 6 weeks. By contrast, children in the Con group were treated with conventional-dose ganciclovir as follows: They were given ganciclovir mixed with $5 \%$ glucose solution through intravenous drip at $5 \mathrm{mg} / \mathrm{kg}$ (12 h/time) for a course of 6 weeks (16).

Basis for efficacy evaluation. The efficacy on the two groups was analyzed, and the number of patients with markedly effective treatment, effective treatment or ineffective treatment was counted, separately. Treatment with the following outcomes was determined as markedly effective: Disappearance of clinical symptoms and signs, and retraction of liver and spleen to normal. Treatment with the following outcomes was determined as effective: Relief of clinical symptoms and signs, and significant retraction of liver and spleen. Treatment with the following outcomes was determined as ineffective: No significant relief of the disease or aggravation for the patient (17). The total number of cases with effective treatment were calculated as: The number of cases with markedly effective treatment + the number of cases with effective treatment.

Determination methods. Venous blood $(5 \mathrm{ml})$ was sampled from each child, and placed in a coagulation promoting tube, and centrifuged at $3,000 \mathrm{x} \mathrm{g}$ and $4^{\circ} \mathrm{C}$ for $10 \mathrm{~min}$ for serum collection, and the miR-UL112-3p expression in the serum was detected using a reverse transcription quantitative PCR (RT-qPCR) assay. Total RNA was extracted from the collected serum with the EasyPure miRNA Kit, and its concentration, purity and integrity were detected by an ultraviolet spectrophotometer and $1 \%$ agarose gel electrophoresis. DNA was extracted using a TIANamp genomic DNA kit (Tianjian Biotechnology Co., Ltd.), and the CMV-DNA level was quantified using a CMV-DNA diagnostic kit (Daan Gene Co., Ltd.). Reverse transcription was carried out to the total RNA using the TransScript ${ }^{\circledR}$ miRNA RT Enzyme Mix and 2X TS miRNA Reaction Mix according to the protocol. PCR amplification was carried out, and the PCR system consisted of $20 \mu \mathrm{l}$ total volume containing $1 \mu \mathrm{l} \mathrm{cDNA}, 0.4 \mu \mathrm{l}$ upstream primers, $0.4 \mu 1$ downstream primers, $10 \mu 1$ 2X TransTaq ${ }^{\circledR}$ Tip 
Table I. Primer sequences.

\begin{tabular}{llc}
\hline Gene & Upstream primer & Downstream primer \\
\hline$m i R-U L 112-3 p$ & 5'-AAGTGACGGTGAGATCCAGGCT-3' & 5'-CCTCCGGATCACATGGTTACTCA-3' \\
U6 & 5'-CTCGCTTCGGCAGCACA-3' & 5'-AACGCTTCACGAATTTGCGT-3' \\
\hline
\end{tabular}

Green qPCR SuperMix, $0.4 \mu \mathrm{l}$ Passive Reference Dye (X50), and $\mathrm{ddH}_{2} \mathrm{O}$ added to adjust the volume. The PCR conditions were: $94^{\circ} \mathrm{C}$ for $30 \mathrm{sec}$, followed by 40 cycles at $94^{\circ} \mathrm{C}$ for $5 \mathrm{sec}$ and $60^{\circ} \mathrm{C}$ for $30 \mathrm{sec}$. Three repeated wells were set for each sample, and the experiment was repeated three times. In the study, U6 was taken as an internal reference and data were analyzed using $2^{-\Delta \Delta c t}(18)$. The total bilirubin (TB), alanine aminotransferase (ALT), and aspartate aminotransferase (AST) were detected using the full automatic biochemical analyzer.

Outcome measures. Primary outcome measures: The Obs group and the Con group were compared in terms of efficacy and changes of liver function indexes (TB, ALT, and AST) and miR-UL112-3p before and after treatment, and Pearson's correlation analysis was carried out to analyze the correlation of serum miR-UL112-3p expression and TB, ALT and AST levels in all children before treatment.

Secondary outcome measures: The clinical baseline data of the two groups were compared (sex, age, course of disease, family size, place of residence, mother's pregnancy age, mother's pregnancy condition, family history of cytomegalovirus infections, and clinical symptoms), and total adverse reactions including gastrointestinal reactions, leucopenia, thrombocytopenia, dizziness, and pruritus of the two groups were also compared after treatment. Additionally, a receiver operating characteristic (ROC) curve was drawn to analyze the predictive value of miR-UL112-3p for cytomegalovirus infections before treatment, andthe AUC, 95\% CI, cut-off point, Youden index, specificity and sensitivity of miR-UL112-3p for predicting efficacy in the two groups were evaluated.

Statistical analysis. The collected data were analyzed using SPSS20.0 (Cabit Information Technology Co., Ltd.), and visualized into required figures using GraphPad Prism 7 (Softhead Software Technology Co., Ltd.). Enumeration data were expressed as usage (\%), and analyzed through the Chi-square test, and expressed by $\chi^{2}$. Measurement data were expressed as the mean \pm standard deviation (mean \pm SD). The relationship among the factors was carried out using the two-way ANOVA, and Bonferroni post hoc test was used for correlation. Pearson's correlation analysis was employed to analyze the correlation between serum miR-UL112-3p expression and TB, ALT and AST levels in all children before treatment. A ROC curve was drawn to evaluate the predictive value of miR-UL112-3p for cytomegalovirus infections before treatment. $\mathrm{P}<0.05$ suggests a significant difference between groups.

\section{Results}

Clinical data. There was no significant difference between the two groups with regard to factors such as sex, age, course of disease, family size, place of residence, mother's pregnancy age, mother's pregnancy condition, family history of cytomegalovirus infections, and clinical symptoms (jaundice, hepatomegaly, and splenomegaly) (all P>0.05) (Table II).

Efficacy. There was no significant difference between the two groups in the number of patients with markedly effective treatment, those with effective treatment, and those without effective treatment, and the total number of patients with markedly effective or effective treatment (all P>0.05) (Table III).

Changes of the liver function before and after treatment. Comparison of the changes of TB, ALT, and AST between the two groups showed that before treatment, there were no significant differences between the Obs group and the Con group

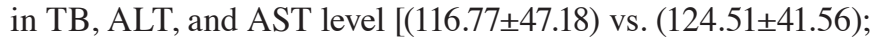
$(175.53 \pm 77.13)$ vs. $(185.37 \pm 71.48)$; $(244.43 \pm 97.20)$ vs. $(251.76 \pm 94.42)]$, while after treatment, the two groups showed decreased TB, ALT, and AST levels, and the Obs group showed significantly lower TB, ALT and AST levels than the Con group [(33.25 \pm 13.73$)$ vs. $(48.35 \pm 16.24)$; $(93.47 \pm 38.36)$ vs.

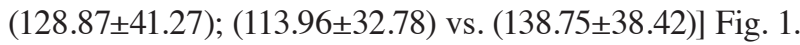

Adverse reactions. The comparison between the two groups in adverse reactions showed that there was no significant difference between the Obs group and the Con group in total adverse reactions including gastrointestinal reactions, leucopenia, thrombocytopenia, dizziness, and pruritus (all $\mathrm{P}>0.05$ ). Table IV.

miR-UL112-3p level and ROC. The comparison between the two groups in the changes of miR-UL112-3p before and after treatment reflected that before treatment, there was no significant difference in miR-UL112-3p expression between the Obs group and the Con group (1.57 \pm 0.62$)$ vs. (1.53 \pm 0.60$)$, while after treatment, both groups showed decreased miR-UL112-3p expression, and the Obs group showed significantly lower miR-UL112-3p expression than the Con group (1.02 \pm 0.39$)$ vs. $(1.21 \pm 0.35)$. We divided the patients in each group into an effective group and an ineffective group, separately. It was found that in the Obs group, the miR-UL112-3p expression in the effective group was significantly lower than that in the ineffective group (1.44 \pm 0.59$)$ vs. $(2.18 \pm 0.39)$, and in the Con group, the miR-UL112-3p expression in the effective group was also significantly lower than that in the ineffective group $(1.41 \pm 0.48)$ vs. $(2.02 \pm 0.32)$. The ROC curve of the Obs group presented that the area under the curve (AUC), specificity, and sensitivity of miR-UL112-3p before treatment in predicting efficacy were $0.866,73.77$ and $84.62 \%$, and its $95 \%$ CI, cut-off point, and Youden index were 0.777-0.955,1.754 and 58.39\%, respectively,and the AUC, specificity, and sensitivity of the 
Table II. Clinical data.

\begin{tabular}{|c|c|c|c|c|}
\hline Variable & Obs group $(n=74)$ & Con group $(\mathrm{n}=67)$ & $t / \chi^{2}$ & P-value \\
\hline Sex & & & 0.028 & 0.867 \\
\hline Male & $43(58.11)$ & $38(56.72)$ & & \\
\hline Female & $31(41.89)$ & $29(43.28)$ & & \\
\hline Age (years) & $3.15 \pm 0.85$ & $3.06 \pm 0.72$ & 0.675 & 0.501 \\
\hline Course of disease (month) & $8.26 \pm 4.34$ & $8.15 \pm 4.18$ & 0.153 & 0.879 \\
\hline No. of family members & & & 0.556 & 0.456 \\
\hline$>3$ & $19(25.68)$ & $21(31.34)$ & & \\
\hline$\leq 3$ & $55(74.32)$ & $46(68.66)$ & & \\
\hline Place of residence & & & 0.151 & 0.698 \\
\hline Urban area & $60(81.08)$ & $56(83.58)$ & & \\
\hline Rural area & $14(18.92)$ & $11(16.42)$ & & \\
\hline Mother' pregnant age & $26.16 \pm 4.63$ & $25.44 \pm 4.37$ & 0.947 & 0.345 \\
\hline Pregnancy & & & 0.794 & 0.373 \\
\hline Multiparity & $49(66.22)$ & 49 (73.13) & & \\
\hline Primiparity & $25(33.78)$ & $18(26.87)$ & & \\
\hline Mother's abnormal pregnancy history & & & 0.059 & 0.808 \\
\hline Yes & $11(14.86)$ & $9(13.43)$ & & \\
\hline No & $63(85.14)$ & $58(86.57)$ & & \\
\hline \multicolumn{5}{|l|}{ Clinical symptoms } \\
\hline Jaundice & $48(64.86)$ & $36(53.73)$ & 1.810 & 0.179 \\
\hline Hepatomegaly & $32(43.24)$ & $25(37.31)$ & 0.513 & 0.474 \\
\hline Splenomegaly & $9(12.16)$ & $8(11.94)$ & 0.002 & 0.968 \\
\hline
\end{tabular}

Table III. Efficacy.

\begin{tabular}{lcccc}
\hline Items & Obs group $(\mathrm{n}=74)$ & Con group $(\mathrm{n}=67)$ & $\chi^{2}$-value & P-value \\
\hline Patients with markedly effective treatment & $19(25.68)$ & $21(31.34)$ & 0.556 & 0.456 \\
Patients with effective treatment & $42(56.75)$ & $36(53.73)$ & 0.130 & 0.718 \\
Patients without effective treatment & $13(17.57)$ & $10(14.93)$ & 0.180 & 0.672 \\
Patients with markedly effective or effective treatment & $61(82.43)$ & $57(85.07)$ & & \\
\hline
\end{tabular}

Table IV. Adverse reactions.

\begin{tabular}{lcccr}
\hline Adverse reactions & Obs group $(\mathrm{n}=74)$ & Con group $(\mathrm{n}=67)$ & $\chi^{2}$-value & P-value \\
\hline Gastrointestinal reactions & $3(4.05)$ & $7(10.45)$ & 2.182 & 0.140 \\
Leucopenia & $4(5.41)$ & $5(7.46)$ & 0.249 & 0.618 \\
Thrombocytopenia & $5(6.76)$ & $7(10.45)$ & 0.615 & 0.433 \\
Dizziness & $2(2.70)$ & $5(1.49)$ & 0.247 & 0.619 \\
Pruritus & $4(5.41)$ & $5(7.46 \%)$ & 0.249 & 0.617 \\
Total adverse reactions & $18(24.32)$ & $25(37.31)$ & 2.799 & 0.094 \\
\hline
\end{tabular}

ROC of the Con group were 0.837, 75.44 and $90.00 \%$, and its 95\% CI, cut-off point, and Youden index were 0.736-0.938, 1.679 and $65.44 \%$, respectively. The AUC of the two groups was $>0.8$, indicating that miR-UL112-3p has good predictive value in therapeutic effect diagnosis (Fig. 2).
Relationship between the miR-UL112-3p expression and liver function indexes. Pearson's correlation analysis was carried out to analyze the relationship between the miR-UL112-3p expression and liver function indexes (TB, ALT, and AST) in all children in the Obs group before treatment, and it was 


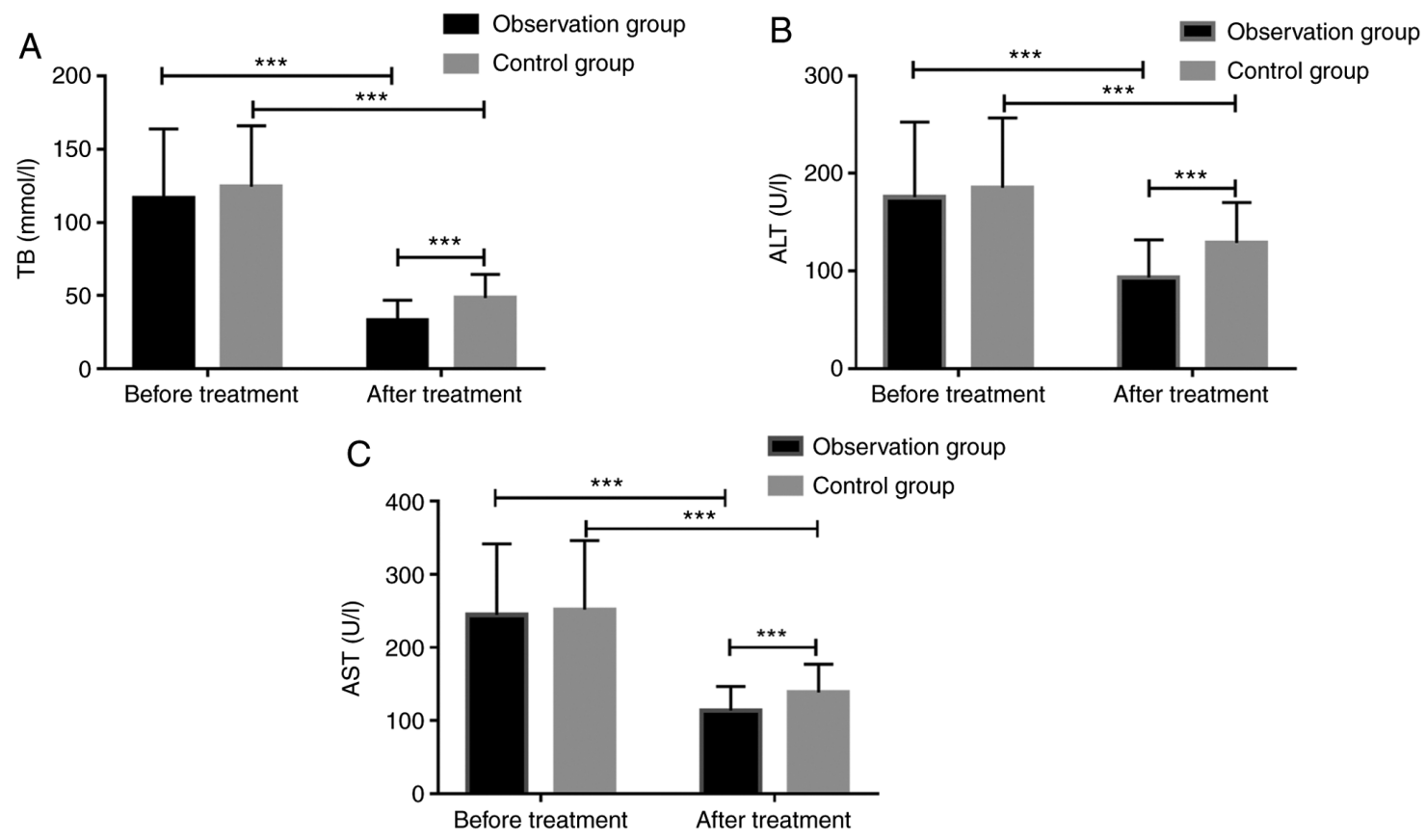

Figure 1. Changes of the liver function before and after treatment. (A) Before treatment, there was no significant difference in TB level between the two groups $(\mathrm{P}>0.05)$, while after treatment, both groups showed decreased TB level, and the TB level in the Obs group was significantly lower than that of the Con group $(\mathrm{P}<0.001)$. (B) Before treatment, there was no significant difference in ALT level between the two groups $(\mathrm{P}>0.05)$, while after treatment, both groups showed a lower ALT level, and the ALT level in the Obs group was significantly lower than that of the Con group $(\mathrm{P}<0.001)$. (C) Before treatment, there was no significant difference in AST level between the two groups ( $\mathrm{P}>0.05$ ), while after treatment, both groups showed a lower AST level, and the AST level in the Obs group was significantly lower than that of the Con group $(\mathrm{P}<0.001) .{ }^{* * *} \mathrm{P}<0.001$.
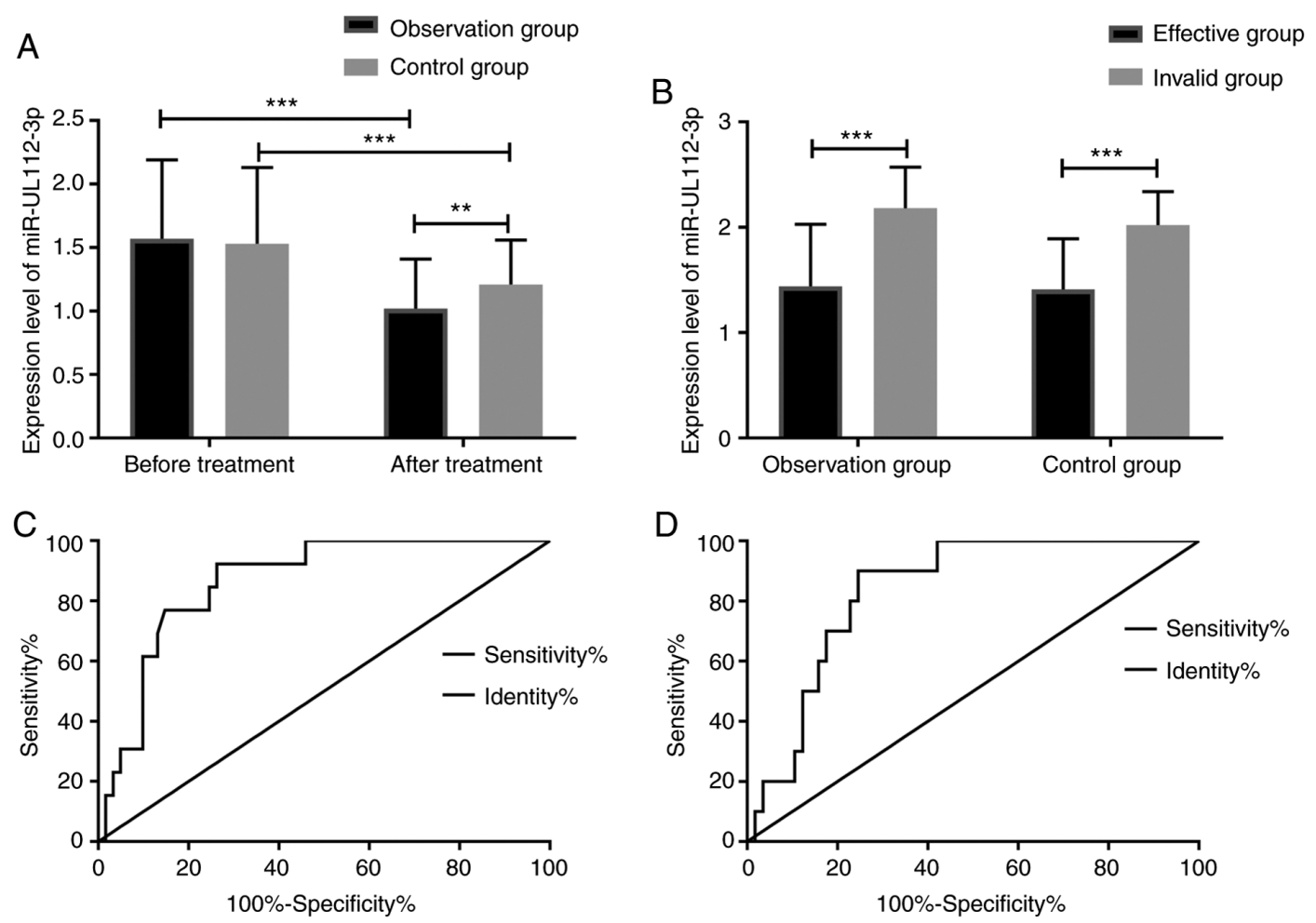

Figure 2. miR-UL112-3p changes and ROC curve for efficacy prediction. (A) Comparison of miR-UL112-3p expression between the observation group and the control group before and after treatment. Before treatment, there was no significant difference in miR-UL112-3p expression between the Obs group and the Con group $(\mathrm{P}>0.05)$, while after treatment, both groups showed a decreased miR-UL112-3p expression, and the decrease in the Obs group was significant $(\mathrm{P}=0.003)$. (B) Before treatment, in the Obs group, the effective group showed a significantly lower miR-UL112-3p expression than the ineffective group $(\mathrm{P}<0.001)$, and in the Con group, the effective group also showed a significantly lower miR-UL112-3p expression than the ineffective group $(\mathrm{P}<0.001)$. $(\mathrm{C})$ The AUC of miR-UL112-3p in predicting efficacy of the Obs group was 0.866, and the 95\% CI thereof was 0.777-0.955. When the cut-off point was $>1.754$, the best specificity and sensitivity were 73.77 and $84.62 \%$, respectively, and the Youden index was $58.39 \%$. (D) The AUC of miR-UL112-3p of the Con group in predicting efficacy was 0.837 , and the $95 \% \mathrm{CI}$ of it was $0.736-0.938$. When the cut-off point was $>1.679$, the best specificity and sensitivity were 75.44 and $90.00 \%$, respectively, and the Youden index was $65.44 \% .{ }^{* *} \mathrm{P}<0.01,{ }^{* * *} \mathrm{P}<0.001$. 

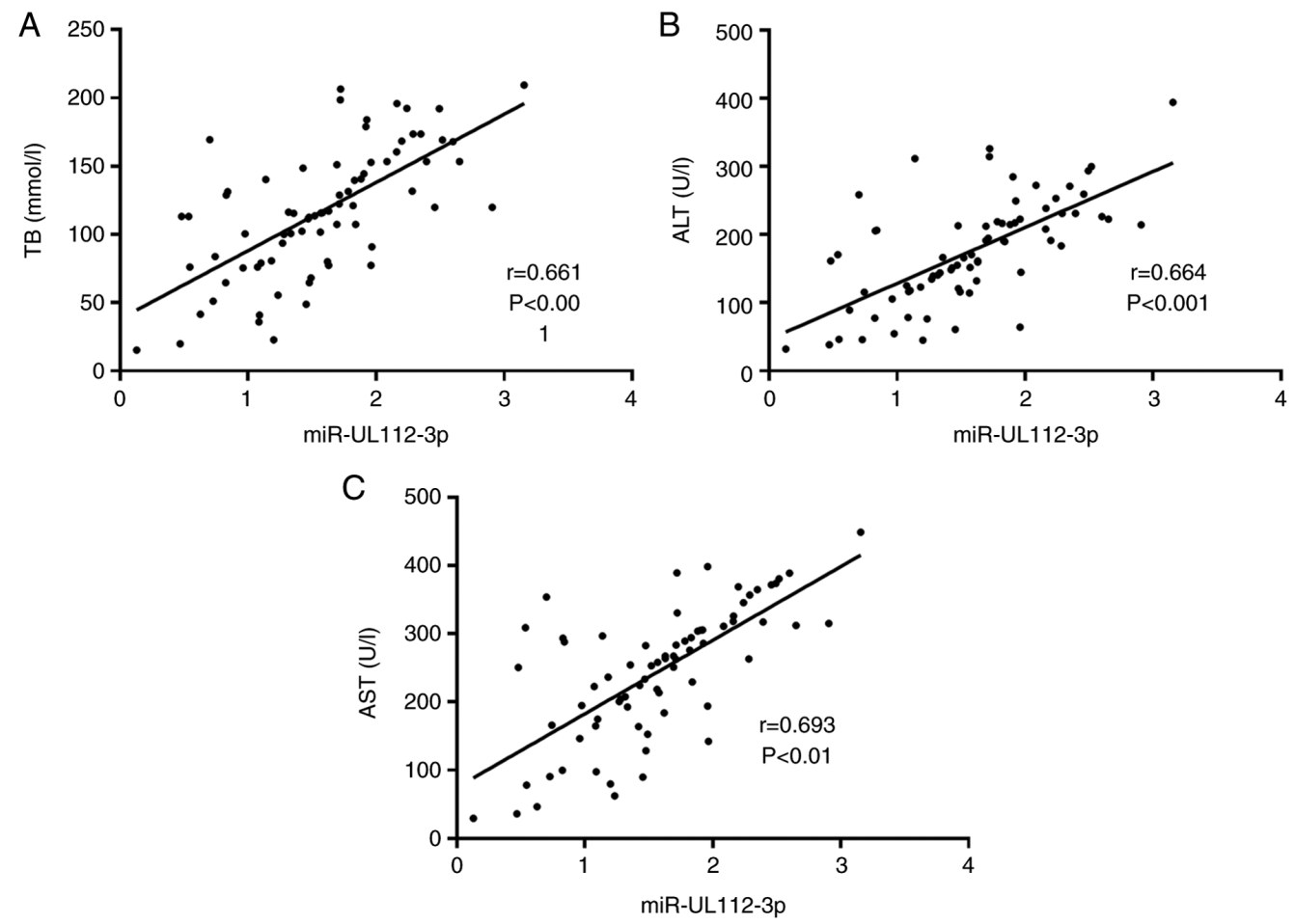

Figure 3. Relationship between the miR-UL112-3p expression and liver function indexes. (A) TB level was positively related with the miR-UL112-3p expression $(r=0.745, P<0.001)$. (B) ALT level was positively related with the miR-UL112-3p expression $(r=0.711, P<0.001)$. (C) AST level was positively related with the miR-UL112-3p expression $(\mathrm{r}=0.723, \mathrm{P}<0.001)$.
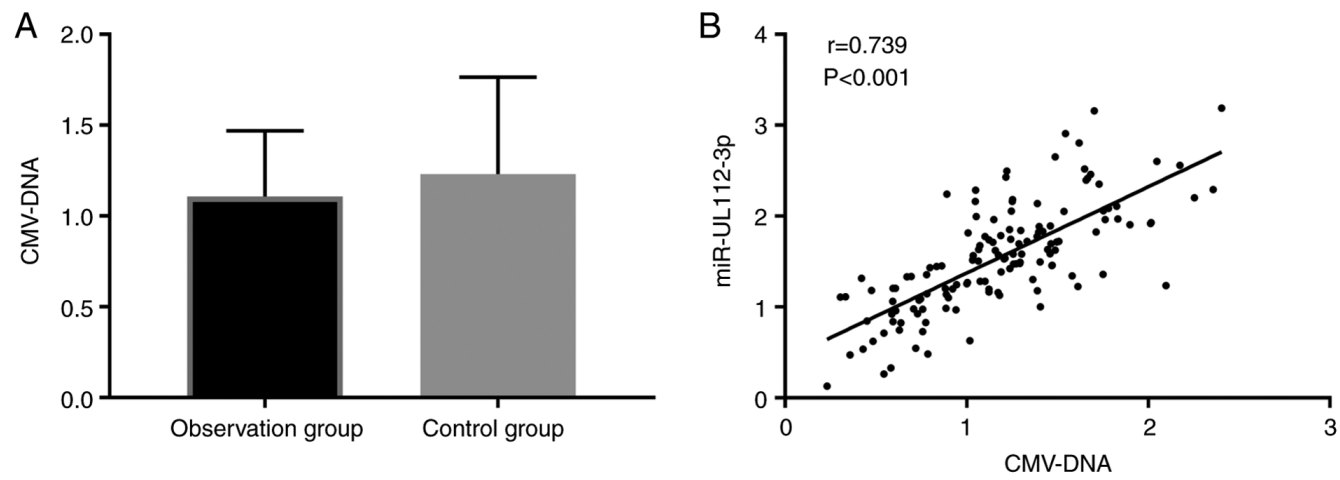

Figure 4. Correlation between the level of CMV-DNA and that of miR-UL112-3p in the two groups. (A) There was no significant difference in CMV-DNA level between the Obs group and the Con group ( $P>0.05)$. (B) Level of CMV-DNA was positively correlated with that of miR-UL112-3p $(r=0.739, \mathrm{P}<0.001)$.

found that miR-UL112-3p was positively correlated with TB, ALT and AST, respectively (Fig. 3).

$C M V-D N A$ level and its correlation with miR-UL112-3p in the two groups. We compared the CMV-DNA level between the two groups after treatment, finding that the CMV-DNA level in the Obs group was lower than that in the Con group $(1.11 \pm 0.36)$ vs. $(1.23 \pm 0.53)$, but the difference was not significant $(\mathrm{P}>0.05)$. We also analyzed the correlation between the level of CMV-DNA and that of miR-UL112-3p in children, finding a positive correlation between them (Fig. 4).

\section{Discussion}

Cytomegalovirus infections, after infecting human body, remain in the precursors of myeloplasts and monocytes, and carry out replicative infection. This infection type can downregulate HLA-I and upregulate Fas ligand to protect itself and promote apoptosis of activated $\mathrm{T}$ cells, thus escaping from the immune process (19). Ganciclovir is effective in the treatment of cytomegalovirus infections; however, it results in unavoidable adverse reactions. Previous studies have reported that half-dose ganciclovir is an effective and safe treatment for children with CMV infections after hematopoietic stem cell transplantation with low CMV antigen level $(16,20)$. Therefore, this study explored whether reducing the dose of ganciclovir affected its efficacy on cytomegalovirus infections in children.

Firstly, we compared the clinical efficacy on the Obs group and that on the Con group, finding that there was no significant difference between the two groups in the number of patients with markedly effective treatment, those with effective treatment, and those without effective treatment, suggesting that 
the efficacy of small-dose ganciclovir is relatively close to that of conventional-dose ganciclovir, and the dose difference has less influence on the efficacy. We also compared the changes of the liver function between the two groups, finding that before treatment, there was no significant difference between the groups in TB, ALT, and AST levels, while after treatment, TB, ALT, and AST levels in the two groups decreased, and those in the Obs group were significantly lower than those in the Con group. Children infected with cytomegalovirus often have higher TB, ALT and AST due to liver damage $(21,22)$. However, with inhibition and control on the virus in children via small-dose ganciclovir, their liver function slowly recovers. It has been previously reported that ganciclovir may affect the liver and kidney function of patients (23). We suspected that small-dose ganciclovir would exert less effect on the liver, making it more effective in improving the liver function.

Furthermore, the adverse reactions in the two groups were compared, showing that the children suffered from adverse reactions including gastrointestinal reactions, leucopenia, thrombocytopenia, dizziness, and pruritus, which have been mentioned in previous studies (24), and there was no significant difference in adverse reactions between the two groups. Although the total adverse reaction rate of the Obs group was lower than that of the Con group, the difference was insignificant.

Subsequently, the miR-UL112-3p expression between the two groups was compared, indicating that before treatment, there was no significant difference between the two groups, while after treatment, both groups showed decreased miR-UL112-3p expression, and the miR-UL112-3p expression in the Obs group was significantly lower than that in the Con group. The increase of miR-UL112-3p expression is often caused by the infection due to lyses of cells by viruses after infection, and ganciclovir can exert an antiviral effect, thus inhibiting the infection of the virus and lowering the miR-UL112-3p expression. We compared miR-UL112-3p expression between the effective group and the ineffective group, and found that in the Obs group and the Con group, the miR-UL112-3p expression in the effective group was significantly lower than that in the ineffective group. This findings suggests prediction of the efficacy on children by analyzing the miR-UL112-3p expression in children prior to treatment. Therefore, we drew a ROC curve of miR-UL112-3p expression in the Obs group and the Con group before treatment, and it was found that the AUC, specificity, and sensitivity of miR-UL112-3p in the ROC curve of the Obs group were 0.866 , 73.77 and $84.62 \%$, respectively, while the AUC, specificity, and sensitivity of the ROC of the Con group were $0.837,75.44$ and $90.00 \%$, respectively. We also found that the AUC of the ROC curve of miR-UL112-3p in each group was $>0.8$, and the AUC of miR-UL112-3p in the Obs group was $>0.85$, indicating that miR-UL112-3p has a good predictive value. It indicates that miR-UL112-3p is a potential prediction indicator for efficacy on children infected with cytomegalovirus.

In a recent study, it was mentioned that the level of serum miR-US25-1-5p in children with cytomegalovirus infections was positively correlated with the levels of elevated serum $\gamma$-glutamyltranspeptidase, direct bilirubin, and total bile acid (25). Finally, we carried out Pearson's correlation analysis to explore the correlation between miR-UL112-3p and liver function indexes in all children before treatment, and it was found that miR-UL112-3p was positively correlated with TB, ALT and AST levels, respectively. Furthermore, miR-UL112-3p is an important miRNA with which cytomegalovirus can escape from the host immune system (26). The increase of miR-UL112-3p expression reflects the activity of cytomegalovirus, and it affects the liver function of patients. In a study by Zhang et al (25) it was identified that miR-UL112-3p expression in infants infected with cytomegalovirus positively correlated with the serum level of bilirubin, indicating that the expression of miR-UL112-3p in infants infected with cytomegalovirus is significantly correlated with the liver function, which also supports our conclusions. Moreover, we verified that the expression of miR-UL112-3p was positively correlated with ALT and AST levels. We also compared the HMV-DNA level in the two groups. It was found that the HMV-DNA level in the Obs group was slightly lower than that in the Con group, but the difference was not significant. Moreover, we found that the level of HMV-DNA in patients was positively correlated with the level of miR-UL112-3p.

However, our study has some limitations. First, subjects enrolled in our study were all patients, and no healthy individual was enrolled; thus, we did not study the differences between the treated children and healthy individuals. Secondly, we identified a particular correlation between miR-UL112-3p and ALT and AST in children through correlation analysis. However, this specific relationship remains to be determined. In addition, we have not tested the CMV-DNA of patients before treatment, thus, the extent of CMV-DNA reduction by treatment with ganciclovir remain to be clarified. Although ganciclovir has always been the first choice for the treatment of cytomegalovirus infections, it only shows moderate antiviral activity, which is not sufficient to completely inhibit virus replication (27). New therapies such as letermovir are under development $(28,29)$, and we hope to compare the efficacy of ganciclovir and those of other therapeutic schemes or combination therapy in subsequent studies.

In conclusion, small-dose ganciclovir can ameliorate the liver function of children, and decrease the expression of miR-UL112-3p. The AUC, specificity, and sensitivity of miR-UL112-3p in the ROC curve of the Obs group were $0.866,73.77$ and $84.62 \%$, respectively, while the AUC, specificity, and sensitivity of the ROC of the Con group were $0.837,75.44$ and $90.00 \%$, respectively. However, the results of the present study remain to be verified and compared against new treatments.

\section{Acknowledgements}

Not applicable.

\section{Funding}

No funding was received.

\section{Availability of data and materials}

The datasets used and/or analyzed during the present study are available from the corresponding author on reasonable request. 


\section{Authors' contributions}

QW, WZ, BW and TH conceived and designed the study, and drafted the manuscript. QW, BW, GQ, FL and DL collected, analyzed and interpreted the experimental data. WZ and TH revised the manuscript for important intellectual content. All authors read and approved the final manuscript.

\section{Ethics approval and consent to participate}

The study was approved by the Ethics Committee of Affiliated Hospital of Weifang Medical University. Signed written informed consents were obtained from the patients and/or guardians.

\section{Patient consent for publication}

Not applicable.

\section{Competing interests}

The authors declare that they have no competing interests.

\section{References}

1. Sun H, Li S, Yan Y, Chen Z, Wang Y, Hao C and Ji W: Associations between patient clinical characteristics and the presence of cytomegalovirus DNA in the bronchoalveolar lavage fluid of children with recurrent wheezing. BMC Infect Dis 18: 458, 2018.

2. Neant N, Klifa R, Bouazza N, Moshous D, Neven B, Leruez-Ville M, Blanche S, Treluyer JM, Hirt D and Frange P: Model of population pharmacokinetics of cidofovir in immunocompromised children with cytomegalovirus and adenovirus infection. J Antimicrob Chemother 73: 2422-2429, 2018.

3. Gentile I,Zappulo E, Riccio MP, Binda S, Bubba L, Pellegrinelli L, Scognamiglio D, Operto F, Margari L, Borgia G and Bravaccio C: Prevalence of congenital cytomegalovirus infection assessed through viral genome detection in dried blood spots in children with autism spectrum disorders. In Vivo 31: 467-473, 2017.

4. Riga M, Korres G, Chouridis P, Naxakis S and Danielides V: Congenital cytomegalovirus infection inducing non-congenital sensorineural hearing loss during childhood; a systematic review. Int J Pediatr Otorhinolaryngol 115: 156-164, 2018.

5. Palma S, Roversi MF, Bettini M, Mazzoni S, Pietrosemoli P, Lucaccioni L, Berardi A and Genovese E: Hearing loss in children with congenital cytomegalovirus infection: An 11-year retrospective study based on laboratory database of a tertiary paediatric hospital. Acta Otorhinolaryngol Ital 39: 40-45, 2019.

6. Ghorbani S, Mahdavi R, Alipoor B, Panahi G, Nasli Esfahani E, Razi F, Taghikhani M and Meshkani R: Decreased serum microRNA-21 level is associated with obesity in healthy and type 2 diabetic subjects. Arch Physiol Biochem 124: 300-305, 2018.

7. Gong YY, Luo JY, Wang L and Huang Y: MicroRNAs regulating reactive oxygen species in cardiovascular diseases. Antioxid Redox Signal 29: 1092-1107, 2018

8. Belarbi Y, Mejhert N, Gao H, Arner P, Rydén M and Kulyté A: MicroRNAs-361-5p and miR-574-5p associate with human adipose morphology and regulate EBF1 expression in white adipose tissue. Mol Cell Endocrinol 472: 50-56, 2018.

9. Ding M, Wang X, Wang C, Liu X, Zen K, Wang W, Zhang CY and Zhang C: Distinct expression profile of HCMV encoded miRNAs in plasma from oral lichen planus patients. J Transl Med 15: 133, 2017.

10. Liang Q, Wang K, Wang B and Cai Q: HCMV-encoded miR-UL112-3p promotes glioblastoma progression via tumour suppressor candidate 3. Sci Rep 7: 44705, 2017.

11. Pan Y, Wang N, Zhou Z, Liang H, Pan C, Zhu D, Liu F, Zhang CY, Zhang Y and Zen K: Circulating human cytomegalovirus-encoded HCMV-miR-US4-1 as an indicator for predicting the efficacy of IFN $\alpha$ treatment in chronic hepatitis B patients. Sci Rep 6: 23007, 2016.
12. Mukhopadhyay R, Roy S, Venkatadri R, Su YP, Ye W, Barnaeva E, Mathews Griner L, Southall N, Hu X, Wang AQ, et al: Efficacy and mechanism of action of low dose emetine against human cytomegalovirus. PLoS Pathog 12: e1005717, 2016.

13. Aryal S, Katugaha SB, Cochrane A, Brown AW, Nathan SD, Shlobin OA, Ahmad K, Marinak L, Chun J, Fregoso M, et al: Single-center experience with use of letermovir for CMV prophylaxis or treatment in thoracic organ transplant recipients. Transpl Infect Dis 21: e13166, 2019.

14. Li J, Pan CW, Zhou GY, Zhuge L, Fang PP, Jin LX, Lin W, Lin XZ and Zheng Y: Studies on the interference of ganciclovir to HCV liver fibrosis. Eur Rev Med Pharmacol Sci 20: 4343-4347, 2016.

15. Kuwahara-Ota S, Chinen Y, Mizuno Y, Takimoto-Shimomura T, Matsumura-Kimoto Y, Tanba K, Tsukamoto T, Mizutani S, Shimura Y, Kobayashi T, et al: Human herpesvirus-6 pneumonitis in a patient with follicular lymphoma following immunochemotherapy with rituximab. Infect Drug Resist 11: 701-705, 2018.

16. Ju HY, Kang HJ, Hong CR, Lee JW, Kim H, Park KD, Shin HY, Park JD, Choi EH, Lee HJ and Ahn HS: Half-dose ganciclovir preemptive treatment of cytomegalovirus infection after pediatric allogeneic hematopoietic stem cell transplantation. Transpl Infect Dis 18: 396-404, 2016.

17. Liu LW, Qian JH, Zhu TW, Zhang YH and Zhu JX: A 5-year retrospective clinical study of perinatal cytomegalovirus infection. Zhongguo Dang Dai Er Ke Za Zhi 18: 99-104, 2016 (In Chinese).

18. Yin CM, Suen WC, Lin S, Wu XM, Li G and Pan XH: Dysregulation of both miR-140-3p and miR-140-5p in synovial fluid correlate with osteoarthritis severity. Bone Joint Res 6: 612-618, 2017.

19. Kasmapour B, Kubsch T, Rand U, Eiz-Vesper B, Messerle M, Vondran FWR, Wiegmann B, Haverich A and Cicin-Sain L: Myeloid dendritic cells repress human cytomegalovirus gene expression and spread by releasing interferon-unrelated soluble antiviral factors. J Virol 92: e01138-17, 2017.

20. Piret J and Boivin G: Clinical development of letermovir and maribavir: Overview of human cytomegalovirus drug resistance. Antiviral Res 163: 91-105, 2019.

21. Ye B and Zhao H: Early abnormal liver enzyme levels may increase the prevalence of human cytomegalovirus antigenaemia after hematopoietic stem cell transplantation. J Int Med Res 45: 673-679, 2017.

22. Nolan N, Halai UA, Regunath H, Smith L, Rojas-Moreno C and Salzer W: Primary cytomegalovirus infection in immunocompetent adults in the United States-A case series. IDCases 10: 123-126, 2017.

23. Ando G, Taguchi K, Enoki Y, Yokoyama Y, Kizu J and Matsumoto K: Evaluation of the expression time of ganciclovir-induced adverse events using JADER and FAERS. Biol Pharm Bull 42: 1799-1804, 2019.

24. Imai S, Yamada T, Kasashi K, Ishiguro N, Kobayashi M and Iseki K: Construction of a flow chart-like risk prediction model of ganciclovir-induced neutropaenia including severity grade: A data mining approach using decision tree. J Clin Pharm Ther 44: 726-734, 2019.

25. Zhang J, Huang Y, Wang Q, Ma Y, Qi Y, Liu Z, Deng J and Ruan Q: Levels of human cytomegalovirus miR-US25-1-5p and miR-UL112-3p in serum extracellular vesicles from infants with HCMV active infection are significantly correlated with liver damage. Eur J Clin Microbiol Infect Dis 39: 471-481, 2020.

26. Landais I, Pelton C, Streblow D, DeFilippis V, McWeeney S and Nelson JA: Human cytomegalovirus miR-UL112-3p targets TLR 2 and modulates the TLR $2 /$ IRAK1/NF $K \mathrm{~B}$ signaling pathway. PLoS Pathog 11: e1004881, 2015.

27. Britt WJ and Prichard MN: New therapies for human cytomegalovirus infections. Antiviral Res 159: 153-174, 2018.

28. Hahn F, Hutterer C, Henry C, Hamilton ST, Strojan H, Kraut A, Schulte U, Schütz M, Kohrt S, Wangen C, et al: Novel cytomegalovirus-inhibitory compounds of the class pyrrolopyridines show a complex pattern of target binding that suggests an unusual mechanism of antiviral activity. Antiviral Res 159: 84-94, 2018.

29. Andronova VL: Modern ethiotropic chemotherapy of human cytomegalovirus infection: Clinical effectiveness, molecular mechanism of action, drug resistance, new trends and prospects. Part 1. Vopr Virusol 63: 202-211, 2018 (In Russian). 